\title{
Pluripotency of induced pluripotent stem cells
}

\author{
Lan Kang and Shaorong Gao*
}

\begin{abstract}
Recent studies have demonstrated that differentiated somatic cells from various mammalian species can be reprogrammed into induced pluripotent stem (iPS) cells by the ectopic expression of four transcription factors that are highly expressed in embryonic stem (ES) cells. The generation of patient-specific iPS cells directly from somatic cells without using oocytes or embryos holds great promise for curing numerous diseases that are currently unresponsive to traditional clinical approaches. However, some recent studies have argued that various iPS cell lines may still retain certain epigenetic memories that are inherited from the somatic cells. Such observations have raised concerns regarding the safety and efficacy of using iPS cell derivatives for clinical applications. Recently, our study demonstrated full pluripotency of mouse iPS cells by tetraploid complementation, indicating that it is possible to obtain fully reprogrammed iPS cells directly from differentiated somatic cells. Therefore, we propose in this review that further comprehensive studies of both mouse and human iPS cells are required so that additional information will be available for evaluating the quality of human iPS cells.
\end{abstract}

Keywords: induced pluripotent stem cells, pluripotency, reprogramming, tetraploid complementation

\section{Introduction}

An iPS cell is induced from a non-pluripotent cell, but possesses pluripotency similar to that of ES cells. Takahashi and Yamanaka (2006) first achieved this landmark breakthrough by reprograming mouse embryonic fibroblasts (MEFs) into this new type of pluripotent stem cell via the ectopic expression of only four transcription factors, namely Oct4, Sox2, Klf4 and c-Myc. This new procedure circumvented the need for an oocyte, which is required by an earlier method of generating customized pluripotent stem cells termed somatic cell nuclear transfer (SCNT)-mediated nuclear reprogramming [1-3]. Since the discovery of iPS cells, the field has attracted a great amount of scientific and public attention because of the undefined mechanism by which the developmental potential of the cells is reverted and the potential for clinical applications using patient specific iPS cells. The generation of iPS cells from individual patients has raised the hope of treatments for numerous degenerative and genetic diseases [4-11].

Unlike normal fertilization or the generation of SCNT-ES cells, the creation of iPS cells is a longer process that results in a heterogeneous mixture of

\footnotetext{
* Correspondence: gaoshaorong@nibs.ac.cn

National Institute of Biological Sciences, NIBS, Beijing, 102206, China
}

cells with various developmental potentials. In the primary culture, iPS cells are usually present together with the original somatic cells, transformed cells and partially reprogrammed cells. Indeed, iPS cells are only approximately $0.1 \%$ to $1 \%$ of the total cells used for reprogramming. Moreover, only very small proportions of these cells are fully reprogrammed based on stringent criteria for evaluating pluripotency. Therefore, it is necessary to establish a molecular standard to distinguish fully reprogrammed iPS cells from those that are partially reprogrammed, especially for human iPS cells that may eventually be used for clinical applications.

In the present review, we will summarize the most recent progress toward understanding the pluripotency of mouse iPS cells at the functional and molecular levels. We anticipate that further studies will be undertaken to improve our understanding of the determination and regulation of pluripotency in human iPS cells.

\section{Differentiation Potential of Stem Cells}

The capacity for differentiation into other cell types under the appropriate conditions is the most important property of early embryonic cells and stem cells. Based on distinct differentiation capabilities, stem cells can be subdivided into pluripotent, multipotent and unipotent stem cells [12]. Only zygotes and the blastomeres of 
early embryos (before the 8-cell stage in mice) during development possess totipotency. Totipotent embryos differentiate into more than 200 types of cells that belong to the three germ layers of development, as well as extraembryonic tissues in vivo, thereby producing new life. After this stage, blastomeres lose totipotency and undergo the first cell fate determination. At the blastocyst stage, a small number of blastomeres develop into the pluripotent inner cell mass (ICM) and the rest differentiate into trophectoderm that forms the extraembryonic tissues and supports embryonic development. The ICM differentiates further into the three germ layers (ectoderm, mesoderm and endoderm) and then into the entire body. This type of developmental capacity is termed pluripotency. Importantly, the ICM can be isolated and maintained in vitro to derive ES cells, which can be maintained in the same pluripotent state as the ICM $[13,14]$. Numerous tissue-specific stem cells, including hematopoietic stem cells, are multipotent and can differentiate into various cell types within the same cell lineage. The spermatogonial stem cell (SSC) is one example of a unipotent tissue-specific stem cell, because SSCs can only differentiate into spermatozoa.

The differentiation potential of stem cells is confirmed by various differentiation assays. Multipotent and unipotent stem cells should have the ability to differentiate into specific cell types after transplantation under the appropriate in vivo conditions or by in vitro culture with the appropriate stimuli. However, it is not practical to differentiate pluripotent stem cells into all of the possible cell types of an organism in vitro, although an embryoid body (EB) that forms the three germ layers can be induced. Subcutaneous transplantation of pluripotent stem cells into an immune deficient mouse produces a teratoma. The formation of teratomas has been used as the most preliminary assay for testing the pluripotency of mouse pluripotent stem cells in vivo. A more stringent assay for testing pluripotency is to generate chimeric mice with a germ line transmission ability. However, the chimera assay may not convincingly represent the full pluripotency of pluripotent stem cells.

Tetraploid blastocyst complementation remains the most stringent assay for testing the pluripotency of pluripotent stem cells. Tetraploid blastocysts are produced via the fusion of 2-cell stage embryos and are developmentally defective by only forming extraembryonic tissues in vivo [15]. Interestingly, this developmental characteristic of tetraploid embryos is exactly the opposite of pluripotent stem cells. As expected, ES cells with full pluripotency compensate for the developmental deficiency of tetraploid embryos, and a full-term organism can be produced from pluripotent stem cells together with extraembryonic tissues derived from tetraploid embryos (Figure 1A) [16,17]. In this manner, ES cells differentiate into all of the various types of fetal cells, tissues and organs, which organize into the organism and truly demonstrate the pluripotency of ES cells. A tetraploid complementation assay may also be considered a type of reconstruction assay similar to the multipotency test for hematopoietic stem cells, but the assay reconstructs the whole fetus instead of only the hematopoietic system (Figure 1B). This information is more useful than in vitro differentiation of stem cells because it can clearly demonstrates that stem cells possess greater potential for differentiation compared that of other stem cell types.

\section{Functional Evaluation of Mouse iPS Cell Pluripotency}

The landmark achievement by Takahashi and Yamanaka revealed that ectopic expression of four transcription factors in differentiated MEFs can induce nuclear reprogramming to form iPS cells with a typical ES cell morphology. These iPS cells express pluripotency genes and produce teratomas following subcutaneous transplantation into immune deficient nude mice. However, live chimeric mice could not be produced by implanting the original iPS cells into normal fertilized embryos [18]. Therefore, the original iPS cells were not fully pluripotent iPS cells. Subsequently, adult chimeras with germ line transmission ability were generated from iPS cells with improved quality [19-21]. However, it remains uncertain whether fully pluripotent iPS cells can be induced because full-term animals could not be produced from iPS cells via tetraploid complementation, even with extensive efforts [21].

To demonstrate fully pluripotent iPS cells, numerous iPS cell lines underwent tetraploid complementation assays, and live pups were finally generated in three independent laboratories including our own [22-24]. After these studies were performed, the data collectively demonstrated that iPS cells are functionally comparable with that of ES cells. Follow-up experiments provided further evidence that iPS cells derived from fetal somatic cells are not the only type of inducible pluripotent cells. Indeed, iPS cells derived from adult somatic cells can also be fully pluripotent [25]. Furthermore, our recent studies have shown that iPS cells that are reprogrammed with only three factors (without c-Myc) can be fully pluripotent, because viable mice can be produced entirely from the iPS cells [26]. However, it should be noted that the success of producing mice consisting of only iPS cells was likely due to the large number of iPS cell lines being examined, and the majority of iPS cell lines that were tested did not produce viable mice.

\section{Differences between Fully Pluripotent iPS Cells and Non- fully Pluripotent iPS Cells}

To further characterize the differences between fully pluripotent iPS cell lines and non-fully pluripotent iPS cell lines, gene expression was compared between a 


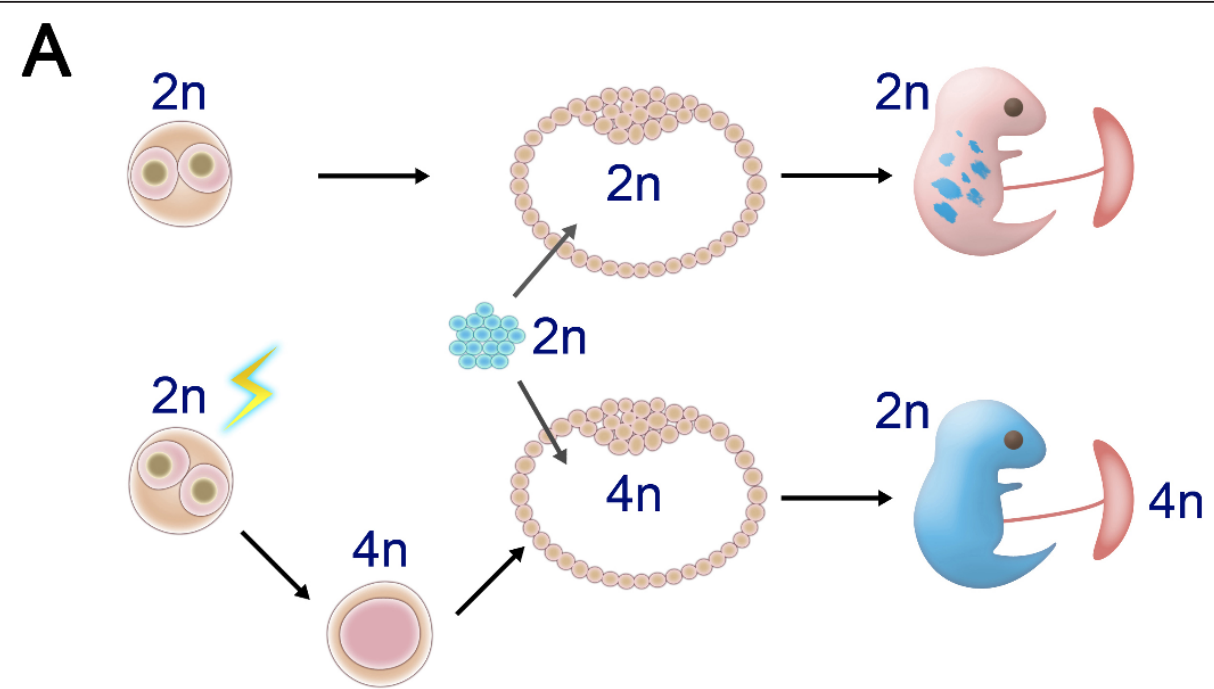

B

\section{Differentiation}
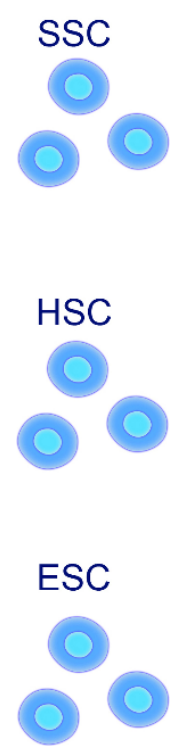
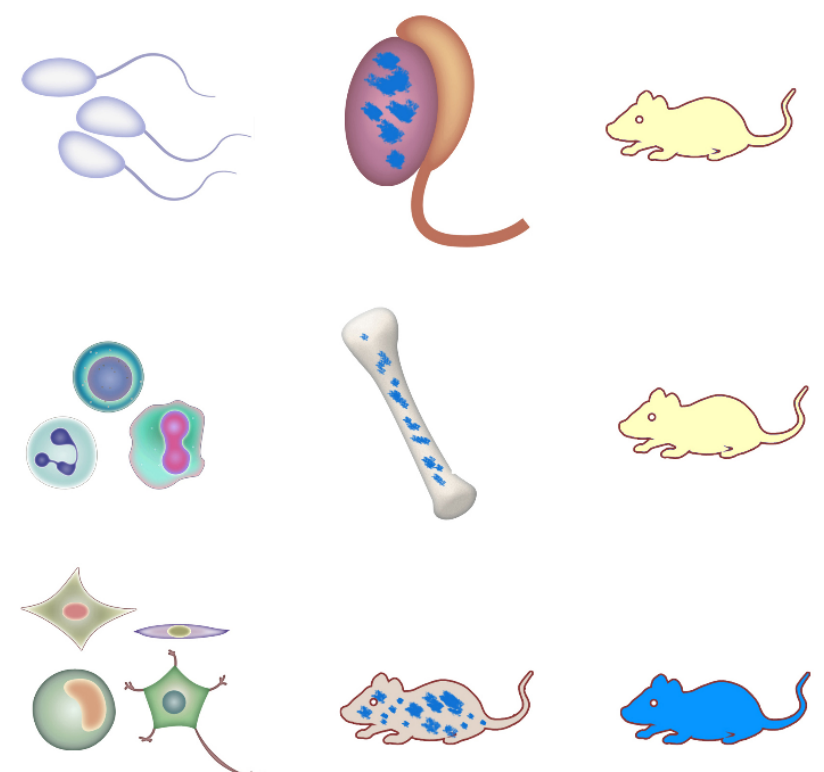

Figure 1 Developmental potential of induced pluripotent stem (iPS) cells. A). Chimera and tetraploid embryo complementation assays for evaluating the pluripotency of pluripotent stem cells. Chimeric mice are produced by aggregation of iPS cells with normal diploid embryos. For tetraploid embryo complementation, the full-term organism is produced by iPS cells, whereas the extraembryonic placenta is derived from a tetraploid embryo that is generated by electric fusion of a 2-cell stage embryo. B). Functional assays for evaluating the differentiation potential of stem cells. Spermatogonial stem cells are unipotent stem cells and can differentiate into sperm as well as contribute toward formation of the testes after transplantation. Hematopoietic stem cells are multipotent stem cells and can differentiate into various hematopoietic cells and contribute toward bone marrow. Moreover, hematopoietic stem cells can reconstruct the entire hematopoietic system of irradiated mice. ES cells are pluripotent stem cells and can theoretically differentiate into all cell types of an organism. Following transplantation into normal diploid blastocysts, ES cells contribute toward the formation of all tissues of the chimera. Furthermore, ES cells can reconstruct the entire organism following a tetraploid embryo complementation assay. 
variety of ES cells and iPS cell lines. A small number of transcripts encoded within the imprinted Dlk1-Dio3 gene cluster on chromosome 12qF1, particularly Glt2 and Rian, are aberrantly silenced in most iPS cell lines that poorly contribute toward chimeras and fail to support the development of iPS cell-derived organisms using tetraploid embryo complementation [25,27]. In contrast, fully pluripotent iPS cell lines exhibit normal expression levels of these genes in this region. Subsequently, the gene expression status of this region has been proposed as a candidate marker for evaluating the quality of iPS cell lines.

However, silencing of the Dlk1-Dio3 gene cluster does not appear to be the only underlying cause of incomplete pluripotency. Although the pluripotency of most iPS cell lines that were tested correlated well with the expression status of these genes, some exceptions existed, in particular Oct4, Sox 2 and Klf4 used to derive iPS cell lines [26]. Moreover, the fact that Gt12 knockout mice are viable challenges the importance of Gtl2 in determining the pluripotency of iPS cells [28]. It has been reported that treatment by a histone deacetylase inhibitor reactivates the silenced Dlk1-Dio3 cluster in partially reprogrammed iPS cells and rescues the ability of iPS cells to support full-term development of iPS cell-derived mice [25]. However, this study remains debatable because the effects of the inhibitor are very complicated. Therefore, we propose that the quality of iPS cells may not be determined by only one gene cluster, and that further comprehensive studies are necessary for discovering additional candidate genes that may synergistically contribute toward the quality of iPS cells. We suggest that a comprehensive comparison of DNA methylation, gene expression and non-coding RNAs using mouse iPS cell lines derived from the same somatic cells with varying developmental potentials would provide a greater understanding of the regulation of pluripotency. Additionally, a sample pool consisting of various of iPS cell lines derived from multiple cell types and derivation strategies with varying genetic backgrounds should be analyzed to reach a consensus (Figure 2).

\section{Molecular Comparison of Human iPS Cells versus ES Cells} Unlike mouse iPS cells, in which pluripotency is tested by tetraploid complementation assay, evaluating the pluripotency of human iPS cells and ES cells is considered much more preliminary. Currently, the most stringent assay for testing the pluripotency of human pluripotent stem cells is teratoma formation, which only evaluates the differentiation potential to form the three germ layers or a small number of specific cell types, but can not indicate full pluripotency. Recently, molecular analyses of human ES cells and iPS cells using genome wide high-throughput assays have allowed more quantitative comparisons to be performed.

By comparing gene expression profiles, studies from several independent groups have suggested that human iPS cells generally resemble human ES cells, but some recurrent differential gene expression signatures have been observed [29-32]. These differences indicate both insufficient silencing of donor cell-specific genes and insufficient induction of ES cell-specific gene expression. Furthermore, this original memory and incomplete reprogramming appear to attenuate upon extended culture because late passage human iPS cells possess a gene expression profile that is more similar to human ES cells. However, these differential gene expression profiles are considered to be stochastic and are affected by the in vitro micro-environment. Upon the sample pool being augmented, the two pluripotent cell types are not consistently distinguished [33,34].

In addition to the transcriptional profiles, the differential methylation of specific CpG islands is suggested to be distinguishable between human iPS cells and ES cells [35]. Variations in CpG methylation, histone modifications and incomplete reprogramming of non-CpG methylation regions that are proximal to centromeres and telomeres in human iPS cells have been observed in genome-wide profiles of DNA methylation at a singlebase resolution [36]. However, Guenther et al. (2010) [33] confirmed that there is little difference between ES cells and iPS cells with respect to genome-wide maps of nucleosomes with histone H3K4 and H3K27 trimethylation, which supports the previous study of H3K4 and H3K27 trimethylation levels in promoter regions [29].

In our opinion, there are several possibilities regarding the cause of these controversies from studies that use similar technologies. First, several studies have suggested that passaging of iPS cells plays an important role in determining the properties of iPS cells $[29,37,38]$. This is understandable because early-passage iPS cell lines may not have completed the reprogramming process. It will be interesting to investigate whether this is true for all iPS cell lines and if so, evaluation of the reprogramming process should be extended to late passages, and the characterization and application of iPS cells should also be standardized with this aspect.

Second, analytical methods and data interpretation greatly influence study conclusions. For example, the threshold used for the calculation of statistical significance can result in biases. Therefore, controversies based on similar datasets suggest that any potential differences between iPS cells and ES cells are very small. Thus far, no specific significant group has been found.

Third, the properties of various iPS cell and ES cell lines are stochastic. Varying conclusions can be drawn because different cell lines, culture conditions and 

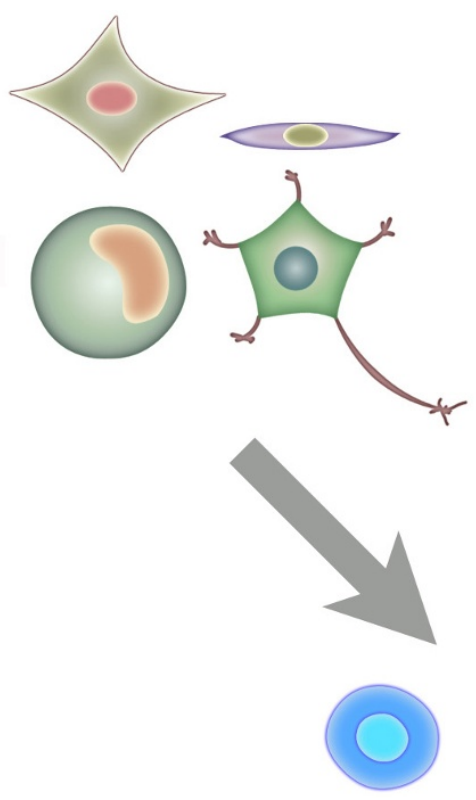

Full
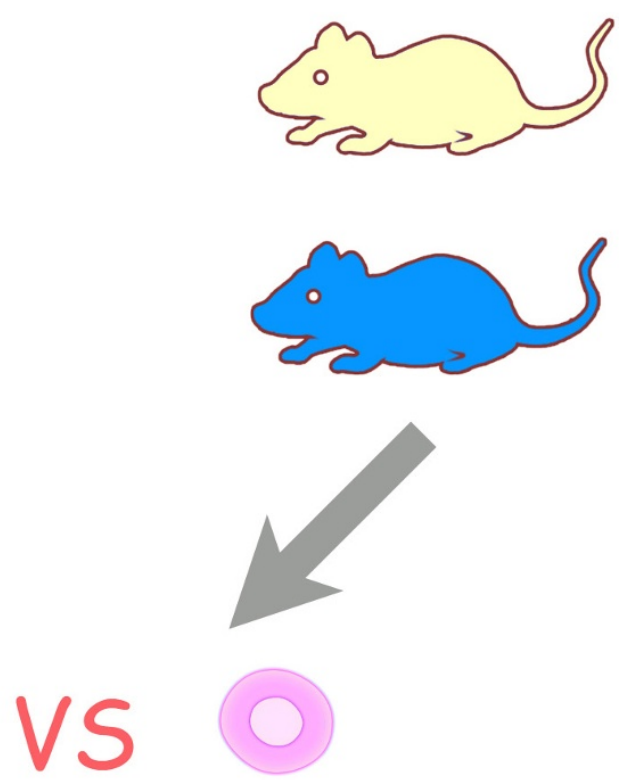

Non-full

\section{mRNA}

Protein

DNA methylation

ncRNA

$\cdots$

\section{Pluripotency}

Figure 2 Molecular analyses of iPS cells. High throughput analyses of mRNA, protein, DNA methylation and non-coding RNA of fully pluripotent and non-fully pluripotent mouse iPS cells would assist our understanding of the pluripotency regulation of iPS cells. iPS cell lines derived from the same somatic cell, but with varying differentiation potentials should be used, and a sample pool consisting of various iPS cell groups derived from multiple cell types should be used to reach a consensus on iPS cell pluripotency.

manipulations are used, and fluctuations can occur within identical microenvironments. Therefore, we suggest increasing the number of tested iPS cell lines to reduce the variation caused by the inadequate comparisons between iPS cell lines.

\section{Treating iPS Cells as ES Cells for Application}

Although the derivation of iPS cells and ES cells is markedly different, there are significant similarities between them. However, a complication that researchers encounter in the application of iPS cells is the heterogenous mixture of cells with various developmental potentials, regardless of which derivation strategy is used. Irrespective of the similarity between fully reprogrammed iPS cells and ES cells, incompletely reprogrammed iPS cells remain present. Therefore, a major consideration for clinical application of iPS cells in the future is to set the minimum criteria to exclude low quality iPS cell lines.

In our opinion, it is not necessary to document the similarity between human iPS cells and ES cells. Human ES cells are also artificial products, and a gold standard is not available for the evaluation of ES cell quality. Clinically, normal cells that can efficiently differentiate into particular cell types are required for regenerative medicine. The use of transdifferentiation for direct conversion of somatic cells into other types of somatic cells has been proposed as an alternative strategy to obtain functional cells for therapy [39-44]. However, the 
efficacy of the resultant cells would need to be confirmed that the shortened telomeres in aged cells are elongated in the converted cells and if not, the usefulness of these cells for cell-based therapy would be unclear. Thus, iPS cells remain the most attractive cell source for clinical purposes.

We propose that the minimum requirements for the clinical use of human iPS cells are: 1) a normal karyotype, because some sub-karyotypic alterations are observed during reprogramming and a normal karyotype is an important factor for ES cell characterization; 2) a normal genotype. Human iPS cell derivation strategies should be improved to preserve the integrity of donor cells, and the method applied without transgenes to include mRNA and protein-mediated induction assisted by small molecules $[45,46]$; 3) the activation of pluripotency networks. This would ensure that iPS cells possess the necessary self-renewal ability and differentiation potential; and 4) a specific differentiation ability. This is the most important criteria and represents the usefulness of iPS cells for specific cell-based therapies.

The epigenetic memory from the original donor cells in incompletely reprogrammed iPS cells would affect the differentiation tendency of early-passage iPS cells $[37,38]$. As previously discussed, iPS cell lines that are stably maintained to late passages should be used. A small number of iPS cell lines have exhibited reduced differentiation efficiencies toward a particular cell type [47], and cell-linespecific differences in DNA methylation and gene expression profiles could lead to an in vitro differentiation propensity [48]. Then, primary exclusion may have a positive effect by increasing the differentiation efficiency, but more convenient examination standards should be applied.

\section{Conclusion}

The direct reprogramming of differentiated somatic cells into iPS cells has enabled us to investigate the molecular events during cell fate choice, as well as the potential clinical use of iPS cells to cure numerous diseases. We have demonstrated the full pluripotency of mouse iPS cells in a functional manner via tetraploid complementation. However, molecular standards for distinguishing iPS cells with various pluripotencies remain to be established. Thus, further comprehensive studies are required for improving our understanding of the regulation of pluripotency within mouse iPS cells. More importantly, similar studies to compare human iPS cells will provide information to establish minimal molecular criteria for evaluating the quality of human iPS cells in the future.

\section{Acknowledgements}

We thank colleagues in our laboratory for their insightful comments. This study was supported by the Ministry of Science and Technology of China (grants 2008AA022311, 2010CB944900 and 2011CB964800).
Authors' contributions

LK and SG wrote and approved the final manuscript.

\section{Competing interests}

The authors declare that they have no competing interests.

Received: 28 December 2011 Accepted: 28 February 2012

Published: 28 February 2012

\section{References}

1. Campbell KH, et al: Sheep cloned by nuclear transfer from a cultured cell line. Nature 1996, 380:64-6

2. Wilmut 1 , et al: Viable offspring derived from fetal and adult mammalian cells. Nature 1997, 385:810-3.

3. Hochedlinger $K$, Jaenisch R: Nuclear transplantation, embryonic stem cells, and the potential for cell therapy. N Engl J Med 2003, 349:275-86.

4. Hanna J, et al: Treatment of sickle cell anemia mouse model with iPS cells generated from autologous skin. Science 2007, 318:1920-3.

5. Dimos JT, et al: Induced pluripotent stem cells generated from patients with ALS can be differentiated into motor neurons. Science 2008, 321:1218-21.

6. Park $\mid \mathrm{H}$, et al: Disease-specific induced pluripotent stem cells. Cell 2008, 134:877-86.

7. Wernig $M$, et al: Neurons derived from reprogrammed fibroblasts functionally integrate into the fetal brain and improve symptoms of rats with Parkinson's disease. Proc Natl Acad Sci USA 2008, 105:5856-61.

8. Ebert $A D$, et al: Induced pluripotent stem cells from a spinal muscular atrophy patient. Nature 2009, 457:277-80.

9. Lee $\mathrm{G}$, et al: Modelling pathogenesis and treatment of familial dysautonomia using patient-specific iPSCs. Nature 2009, 461:402-6.

10. Raya A, et al: Disease-corrected haematopoietic progenitors from Fanconi anaemia induced pluripotent stem cells. Nature 2009, 460:53-9.

11. Soldner F, et al: Parkinson's disease patient-derived induced pluripotent stem cells free of viral reprogramming factors. Cell 2009, 136:964-77.

12. Jaenisch R, Young R: Stem cells, the molecular circuitry of pluripotency and nuclear reprogramming. Cell 2008, 132:567-82.

13. Evans MJ, Kaufman MH: Establishment in culture of pluripotential cells from mouse embryos. Nature 1981, 292:154-6.

14. Thomson JA, et al: Embryonic stem cell lines derived from human blastocysts. Science 1998, 282:1145-7.

15. Tarkowski AK, et al: Development of cytochalasin in B-induced tetraploid and diploid/tetraploid mosaic mouse embryos. J Embryol Exp Morphol 1977, 41:47-64.

16. Nagy A, et al: Embryonic stem cells alone are able to support fetal development in the mouse. Development 1990, 110:815-21.

17. Nagy A, et al: Derivation of completely cell culture-derived mice from early-passage embryonic stem cells. Proc Natl Acad Sci USA 1993, 90:8424-8.

18. Takahashi K, Yamanaka S: Induction of pluripotent stem cells from mouse embryonic and adult fibroblast cultures by defined factors. Cell 2006, 126:663-76.

19. Maherali N, et al: Directly reprogrammed fibroblasts show global epigenetic remodeling and widespread tissue contribution. Cell Stem Cell 2007, 1:55-70.

20. Okita $\mathrm{K}$, et al: Generation of germline-competent induced pluripotent stem cells. Nature 2007, 448:313-7.

21. Wernig $M$, et al: In vitro reprogramming of fibroblasts into a pluripotent ES-cell-like state. Nature 2007, 448:318-24

22. Boland MJ, et al: Adult mice generated from induced pluripotent stem cells. Nature 2009, 461:91-4.

23. Kang $L$, et al: iPS cells can support full-term development of tetraploid blastocyst-complemented embryos. Cell Stem Cell 2009, 5:135-8.

24. Zhao $X Y$, et al: iPS cells produce viable mice through tetraploid complementation. Nature 2009, 461:86-90.

25. Stadtfeld $M$, et al: Aberrant silencing of imprinted genes on chromosome $12 \mathrm{qF} 1$ in mouse induced pluripotent stem cells. Nature 2010, 465:175-81.

26. Kang $L$, et al: Viable mice produced from three-factor induced pluripotent stem (iPS) cells through tetraploid complementation. Cell Res 2011, 21:546-9.

27. Liu L, et al: Activation of the imprinted Dlk1-Dio3 region correlates with pluripotency levels of mouse stem cells. J Biol Chem 2010, 285:19483-90. 
28. Takahashi N, et al: Deletion of Gt12, imprinted non-coding RNA, with its differentially methylated region induces lethal parent-origin-dependent defects in mice. Hum Mol Genet 2009, 18:1879-88.

29. Chin MH, et al: Induced pluripotent stem cells and embryonic stem cells are distinguished by gene expression signatures. Cell Stem Cell 2009, 5:111-23.

30. Marchetto MC, et al: Transcriptional signature and memory retention of human-induced pluripotent stem cells. PLOS ONE 2009, 4:e7076.

31. Chin MH, et al: Molecular analyses of human induced pluripotent stem cells and embryonic stem cells. Cell Stem Cell 2010, 7:263-9.

32. Ghosh Z, et al: Persistent donor cell gene expression among human induced pluripotent stem cells contributes to differences with human embryonic stem cells. PLOS ONE 2010, 5:e8975.

33. Guenther $M G$, et al: Chromatin structure and gene expression programs of human embryonic and induced pluripotent stem cells. Cell Stem Cell 2010, 7:249-57.

34. Newman AM, Cooper JB: Lab-specific gene expression signatures in pluripotent stem cells. Cell Stem Cell 2010, 7:258-62.

35. Doi A, et al: Differential methylation of tissue- and cancer-specific CpG island shores distinguishes human induced pluripotent stem cells, embryonic stem cells and fibroblasts. Nat Genet 2009, 41:1350-3.

36. Lister $\mathrm{R}$, et al: Hotspots of aberrant epigenomic reprogramming in human induced pluripotent stem cells. Nature 2011, 471:68-73.

37. Kim $\mathrm{K}$, et al: Epigenetic memory in induced pluripotent stem cells. Nature 2010, 467:285-90.

38. Polo JM, et al: Cell type of origin influences the molecular and functional properties of mouse induced pluripotent stem cells. Nat Biotechnol 2010, 28:848-55.

39. Weintraub $\mathrm{H}$, et al: Activation of muscle-specific genes in pigment, nerve, fat, liver, and fibroblast cell lines by forced expression of MyoD. Proc Natl Acad Sci USA 1989, 86:5434-8.

40. Zhou $Q$, et al: In vivo reprogramming of adult pancreatic exocrine cells to beta-cells. Nature 2008, 455:627-32.

41. Hanna JH, et al: Pluripotency and cellular reprogramming: facts, hypotheses, unresolved issues. Cell 2010, 143:508-25.

42. leda $M$, et al: Direct reprogramming of fibroblasts into functional cardiomyocytes by defined factors. Cell 2010, 142:375-86.

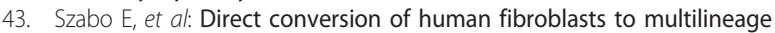
blood progenitors. Nature 2010, 468:521-6.

44. Vierbuchen $T$, et al: Direct conversion of fibroblasts to functional neurons by defined factors. Nature 2010, 463:1035-41.

45. Kim D, et al: Generation of human induced pluripotent stem cells by direct delivery of reprogramming proteins. Cell Stem Cell 2009, 4:472-6.

46. Warren $L$, et al: Highly efficient reprogramming to pluripotency and directed differentiation of human cells with synthetic modified mRNA. Cell Stem Cell 2010, 7:618-30.

47. Hu BY, et al: Neural differentiation of human induced pluripotent stem cells follows developmental principles but with variable potency. Proc Natl Acad Sci USA 2010, 107:4335-40.

48. Bock C, et al: Reference Maps of Human ES and iPS Cell Variation Enable High-Throughput Characterization of Pluripotent Cell Lines. Cell 2011, 144:439-52.

doi:10.1186/2049-1891-3-5

Cite this article as: Kang and Gao: Pluripotency of induced pluripotent stem cells. Journal of Animal Science and Biotechnology 2012 3:5.

\section{Submit your next manuscript to BioMed Central and take full advantage of:}

- Convenient online submission

- Thorough peer review

- No space constraints or color figure charges

- Immediate publication on acceptance

- Inclusion in PubMed, CAS, Scopus and Google Scholar

- Research which is freely available for redistribution

Submit your manuscript at www.biomedcentral.com/submit
Biomed Central 